\title{
A CASE REPORT OF INTRACRANIAL VERTEBRAL-BASILAR AR- TERY HYPOPLASIA PRESENTING WITH EPISODIC DIZZINESS
}

\author{
JIANN JY CHEN ${ }^{1,2}$ and DEM LION CHEN ${ }^{3}$ \\ ${ }^{1}$ Department of Otorhinolaryngology, Taoyuan General Hospital, Taoyuan, Taiwan, Province of China \\ ${ }^{2}$ Department of Medical Imaging, Taipei Medical University-Shuang Ho Hospital, Taipei, Taiwan, Province \\ of China ${ }^{3}$ G-Home Otorhinolaryngologic Clinic, Kaohsiung, Taiwan, Province Of China
}

Corresponding Author: Dr. Chen, Dem-Lion

Conflict of Interest: None declared

\section{SUMMARY}

Basilar artery hypoplasia (BAH) is usually accompanied with unilateral vertebral artery hypoplasia (VAH); however, BAH with bilateral VAHs composing vertebral-basilar artery hypoplasia (VBAH) is indeed a rare curiosity. A 61-year-old woman presented with episodic dizziness for ten years. It accompanied with headache, bilateral tinnitus and blurred vision. Timeof-flight magnetic resonance angiogram with $\mathrm{T} 2$ true fast imaging in steady state precession confirmed the VBAH, rather than a stenosis. Symptoms subsided after daily oral aspirin and life-style change has been recommended for three months. The following one year was uneventful, and hearing improved. Finally, we conclude that the episodic dizziness with sensori-neural hearing impairment might attribute to the VBAH. It appears that life-style change was the main therapy and the antiplatelet was simply a supplementary one.

Key words: aspirin, sensori-neural hearing impairment, vertebral artery, basilar artery, hypoplasia.

\section{INTRODUCTION}

Vertebral artery hypoplasia (VAH) is defined that vertebral artery (VA) is less than $2.0 \mathrm{~mm}$ in diameter by time-of flight magnetic resonance angiogram (TOF MRA). ${ }^{1}$ Besides, basilar artery hypoplasia (BAH) is generally considered if the basilar artery (BA) is less than the common range $(3.0 \mathrm{~mm} \sim 5.5 \mathrm{~mm})$ in diameter, usually incidentally identified in a sufferer with transient ischemia attack or ischemic stroke. ${ }^{2,3} \mathrm{BAH}$ is usually accompanied with unilateral VAH; however, BAH with bilateral VAHs composing vertebral-basilar artery hypoplasia (VBAH) is indeed a rare curiosity, but it has not yet been documented any objective criteria. We incidentally met such a case in 2008 , and report it herein.

\section{CASE REPORT}

A 61-year-old female cleaner with a height of $162 \mathrm{~cm}$, a weight of $68 \mathrm{~kg}$ and a body mass index of $25.9 \mathrm{~kg} / \mathrm{m}^{2}$, did not have hypertension or any heart disease, but has
Email: jiannjy@yahoo.com.tw

regularly taken medication for type II diabetic mellitus for over twenty years. She has been bothered with episodic dizziness once per month since 1998, but up to ten times per day since 2005. Sudden exertion or emotional stress would induce several seconds' attack, accompanied with headache, bilateral tinnitus and blurred vision, rather than syncope, paraesthesia, ataxia, dysarthria, diplopia or other neurologic signs.

When seen in July 2008 the blood pressure was 133/67 $\mathrm{mmHg}$ with a heart rate of $80 / \mathrm{min}$. Physical examinations were generally normal, but a finger-to-nose tracing test showed bilaterally unsmooth; besides, tests for Romberg and tandem gait showed staggering. Hyperventilation (20 deep respirations) and a squat-to-stand test provoked the same dizziness. The orthostatic hypotension test was negative. A pure tone audiometry showed bilateral symmetrical high-tone hearing loss (Figure 1a). The short increment sensitivity indices over $1,000 \mathrm{~Hz}, 2,000 \mathrm{~Hz}$ and $4,000 \mathrm{~Hz}$ were all $0 \%$ in the both ears, but the speech discrimination scores were both $85 \%$.

A colour-coded duplex ultrasonogram showed the bilateral external carotid arteries were mildly atherosclerotic, and the intracranial VAs had more velocity with less volume than the extracranial VAs (Table 1). Magnetic resonance imaging, including $\mathrm{T} 1$, T2, diffusionweighted imaging and apparent diffusion coefficient map, did not show any remarkable lesion, but TOF MRA and T2 true fast imaging in steady state precession (T2 true FISP) demonstrated that (1) the bilateral posterior communicating arteries were absent, and (2) the BA and the bilateral intracranial VAs were hypoplastic (Figure 2a, 2b). All blood studies were within normal range. An electrocardiogram, a 24 hours' Holter and an electroencephalogram did not show any abnormality.

An oral antiplatelet, aspirin $100 \mathrm{mg}$, daily was recommended; besides, she was advised to change the lifestyle, such as to warm up before exertion and to avoid over fatigue. 
Then, the symptoms recurred less and less gradually. Three months later, symptom subsided, so aspirin was halted. Over the following one year, she simply took medication for type II diabetic mellitus and did not take other symptomatic controlling medication, and symptom did not recur any more. Pure tone audiometry showed improving bilateral hearing improved (Fig. 1b). The short increment sensitivity indices over $1,000 \mathrm{~Hz}$, $2,000 \mathrm{~Hz}$ and $4,000 \mathrm{~Hz}$ were all $0 \%$ in the both ears. The speech discrimination scores were both $100 \%$.

\section{DISCUSSION}

Due to negative orthostatic hypotension test, diabetic autonomic neuropathy was unlikely causative. Although prolonged hyperventilation or sudden stand following prolonged squat usually causes dizziness or other discomfort symptoms in a normal healthy person, the result is considered of positive significance if the discomfort symptoms are the same as that bothering the examinee. Because the patient had a positive hyperventilation test and a positive squat-to-stand test, the episodic dizziness with headache, tinnitus and blurred vision was attributable to cerebral hemodynamic decrease or cerebral vasoconstriction. ${ }^{4,5}$ The phenomenal finding of colour-coded duplex ultrasonogram (Table 1) warned us of a vertebral-basilar artery disease. Although digital subtraction angiogram is a gold standard for a vascular disease, ${ }^{6}$ it has a small morbidity associated with mortality; besides, it is considered extravagant to apply this study to a sufferer with simply episodic dizziness without any neurologic focal signs. TOF MRA, much cheaper and safer than digital subtraction angiogram, is currently preferred for the kind of patient at our hospital. In the patient, the TOF MRA with T2 true FISP confirmed the intracranial VBAH (Figure 2a, 2b) rather than a vertebral-basilar artery stenosis.

Table 1 Colour-coded duplex ultrasonogram (EnVisor, Philips, USA) of vertebral-basilar artery

\begin{tabular}{|l|c|c|c|c|c|}
\hline & \multicolumn{2}{|c|}{ Extracranial } & & \multicolumn{3}{|c|}{ Intracranial } \\
\cline { 1 - 1 } & $\begin{array}{c}\text { Right } \\
\text { VA }\end{array}$ & $\begin{array}{c}\text { Left } \\
\text { VA }\end{array}$ & $\begin{array}{c}\text { Right } \\
\text { VA }\end{array}$ & $\begin{array}{c}\text { Left } \\
\text { VA }\end{array}$ & BA \\
\hline Diameter (mm) & 3.39 & 3.50 & & & \\
\hline $\begin{array}{l}\text { Average veloc- } \\
\text { ity (cm/sec) }\end{array}$ & 15.8 & 16.9 & 22.4 & 29.8 & 19.8 \\
\hline $\begin{array}{l}\text { Average flow } \\
\text { (ml/min) }\end{array}$ & 85.6 & 97.6 & & & \\
\hline $\begin{array}{l}\text { Resistance in- } \\
\text { dex }\end{array}$ & 0.46 & 0.61 & 0.49 & 0.55 & 0.63 \\
\hline $\begin{array}{l}\text { Pulsatibility } \\
\text { index }\end{array}$ & 1.04 & 1.71 & 0.68 & 0.77 & 0.84 \\
\hline
\end{tabular}

$\mathrm{VA}=$ vertebral artery; $\mathrm{BA}=$ basilar artery
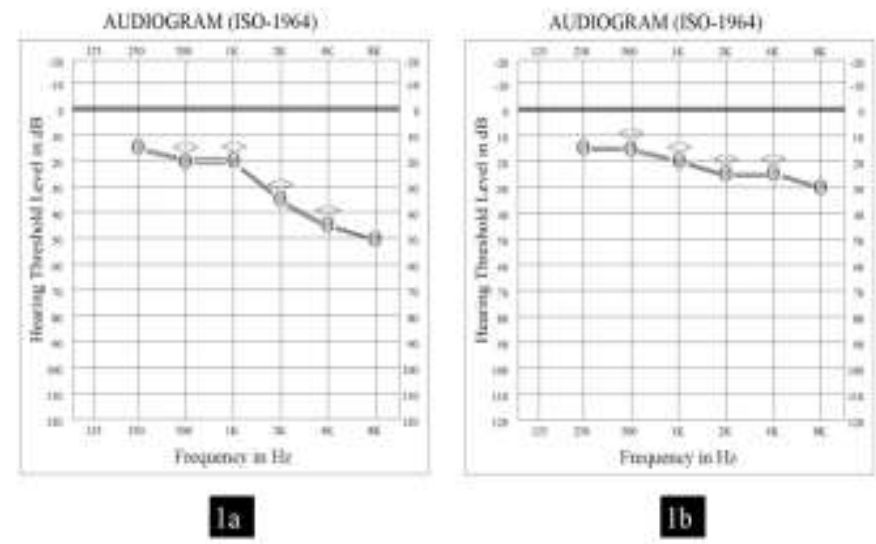

Figure 1a The first pure tone audiometry shows the average hearing threshold between $500 \mathrm{~Hz}$ and $8,000 \mathrm{~Hz}$ are bilaterally $30.8 \mathrm{~dB}$ hearing level (HL). 1b: the follow-up pure tone audiometry shows the average hearing threshold are bilaterally $21.7 \mathrm{dBHL}$. $\mathrm{O}=$ right-ear air conduction; $\times=$ left-ear air conduction; <=right-ear bone conduction; >=left-ear bone conduction.
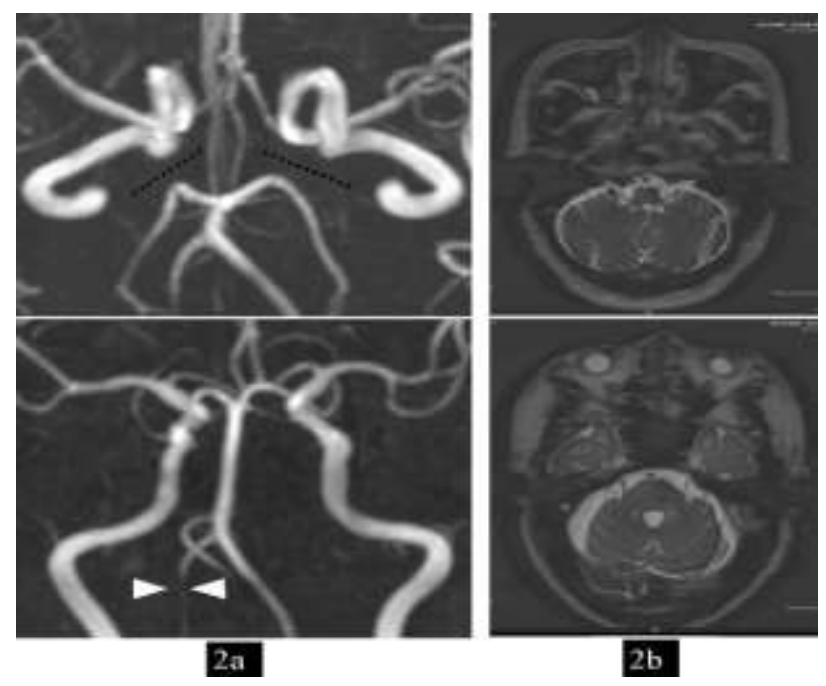

Figure 2a: The TOF MRA (TR/TE/excitation: 27/6/1) reveals that the circle of Willis has posterior defects (dotting lines). Besides, the BA (diameter: $2.5 \mathrm{~mm}$ ), the right intracranial VA (diameter: $1.4 \mathrm{~mm}$ ) and the left intracranial VA $(1.6 \mathrm{~mm})$ are hypoplastic, and there is even a segment of little flow in the left intracranial VA (pincer arrowheads). Figure 2b: the axial T2 true FISP (TR/TE/excitations: 5,000/126/1) reveals that the external diameters of the BA, the right VA and the left VA were respectively $3.4 \mathrm{~mm}, 2.3 \mathrm{~mm}$ and $2.8 \mathrm{~mm}$.

We suggest a certain trigeminal artery did not degenerate as scheduled during bilateral longitudinal neural arteries were fusing medially in the fifth embryologic week. 
The function and development of vertebral-basilar artery was thereby limited, and the VBAH remained after the trigeminal artery degenerated. ${ }^{2}$ Then, age-related atherosclerosis gradually restricted the compliance of the VBAH. Sudden exertion or emotional stress would incur a paradoxical cerebral vasoconstriction and the following transient hemodynamic decrease of her VBAH. ${ }^{7}$

The episodic symptoms occurred in presence of injuries of the poster-circulation territory. Although the magnetic resonance imaging did not show any abnormality, tests for finger-to-nose tracing, Romberg and tandem gait showed abnormalities, implicating there were vestibular, brainstem and cerebellar impairments. Besides, bilateral high-tone hearing loss and speech discrimination scores of $85 \%$ demonstrated both retrocochlear auditory neuropathies, and short increment sensitivity indices of $0 \%$ excluded any cochleopathy.

We could do nothing for the VBAH, and there has not yet been any standard medication for VBAH in the literature, so symptomatic control was empirically recommended. Because vertebral-basilar artery is theoretically contributable to vertebral-basilar insufficiency, 2 we empirically try some medication to reduce the vascular resistance of VBAH. However, anticoagulants or thrombolytic medication are not recommended for such a patient without any ischemic stroke, so daily oral aspirin was an empirically practical strategy for her; besides, she was advised to change the life-style change to avoid any symptomatic induction.

Over the following three months, she regularly took oral aspirin, which is controversial to have ototoxicity. ${ }^{8}$ ${ }^{9}$ Over the following year, she simply took medication for type II diabetic mellitus and did not take aspirin, and it was uneventful.

The follow-up pure tone audiometry and speech discrimination score revealed that the hearing improved
(Figure 1b), explicating recovery of brainstem auditory pathway.

Finally, we conclude that the episodic dizziness with sensori-neural hearing impairment might attribute to recurrent hemodynamic decrease of the VBAH. Although the hearing improved and the dizziness relieved, it did not entirely ascribe the success to aspirin, and it appears that life-style change was the main therapy and the antiplatelet was simply a supplementary one.

\section{REFERENCE}

1. Chuang YM, Huang YC, Hu HH, Yang CY. Toward a further elucidation: role of vertebral artery hypoplasia in acute ischemic stroke. Eur Neurol 2006;55:193-197.

2. Seemant C, Timonthy L, Chen W. Ischemia in the territory of a hypoplastic vertebrobasilar system. Neurology 1999;52:980-983.

3. Turgut N, Pekindil G, Utku U, Celik Y, Şiengün S. Isolated hypoplasia of distal basilar artery: clinical and imaging findings. Yeni Symposium 2004;42:121-125.

4. Savitz SI, Caplan LR. Vertebrobasilar disease. $N$ Engl J Med 2005;352:2618-2626.

5. Vinik AI, Mitchell BD, Maser RE, Freeman R. Diabetic autonomic neuropathy. Diabetes Care 2003;26:1553-1579.

6. Cloud GC, Markus HS. Diagnosis and management of vertebral artery stenosis. $Q \mathrm{~J} \mathrm{Med}$ 2003;96:27-34.

7. Chuang YM, Hu HH, Pan PJ. Cerebral syncope: insights from Valsalva maneuver. Eur Neurol 2005;54:98-102.

8. Koren G. Hearing loss in a woman on aspirin: the silent pharmacokinetic parameter. Ther Drug Monit 2009;31:1-2.

9. Chen Y, Huang WG, Zha DJ, Qiu JH, Wang JL, Sha SH, Schacht J. Aspirin attenuates gentamicin ototoxicity: from the laboratory to the clinic. Hear Res 2007;226:178-182. 